\title{
A Sobel-TV based Hybrid Model for De-noising Remote Sensing Image with Gaussian and Salt-pepper Noise
}

\author{
TU Jihui ${ }^{1}$, Zheng Jiang ${ }^{2}$ and $\mathrm{Wu}$ Xiaodong ${ }^{2}$ \\ ${ }^{1}$ Electronics \& Information School of Yangtze University, Jingzhou, Hubei 434023, \\ China \\ ${ }^{2}$ Powerchina kunming engineering corporation CO., LTD, Kunming, Yunnan 650051, \\ China \\ Igreen666@126.com, ${ }^{2}$ hhuwxd@126.com, ${ }^{2542239616 @ q q . c o m ~}$
}

\begin{abstract}
The pre-processed remote sensing images are often polluted by Gaussian and salt-pepper noises. In order to solve this problem, a Sobel-TV based hybrid model is proposed to de-noise the pre-processed remote sensing images. It uses TV model to de-noise and uses Sobel algorithm to control smoothness of the image' edge. This proposed method will not only efficiently remove image noise but also simultaneously reserves detail information such as edge and texture. Experimental results show the proposed algorithm achieves better SNR and SSIM compared with other methods. In terms of visual quality, the proposed algorithm can remove the noise of the images and preserve more details, which is important value to preprocess remote sensing image.
\end{abstract}

Keywords: TV Model; Sobel algorithm; Sobel-TV Model; Image Denoising

\section{Introduction}

Remote sensing imaging has been a powerful technique for exploring and obtaining knowledge of the world. With the rapid development of remote sensing technology, the highresolution remote sensing images have been applied to various fields which demand the everincreasing quality for remote sensing images. Satellite or aerial images are inevitably corrupted by various noises so that noise seriously affects the analysis and understanding for remote sensing image. Noise is generated by many factors, such as thermal effects, sensor saturation, quantization errors and transmission errors, etc. So remote sensing imagery denoising is a key component in the pre-processing and interpretability of the image. Noise analyzing, estimation and filter of remote sensing image is a hot issue of its processing and application, which has been attracting the attention of remote sensing applications [1-2]. A lot of studies have shown that noise of remote sensing image is the superposition of gauss noise and salt and pepper noise. The smoothing and denoising of remote sensing image contain two parts: firstly, it is effective to remove noise in the image; secondly, it is to retain or enhance the inherent feature of remote sensing image, such as edge and texture features. Although the traditional Gaussian and median filter algorithm is simple and easy to implement, but it is hard to meet the requirements of these two aspects. In recent years, image denoising algorithms based on what is called the Total Variation (TV) model which attracts much many research's attention [3-9]. TV denoising is an approach for noise reduction developed so as to preserve sharp edges in the image. It has faster diffusion in the direction parallel to the edge, while the diffusion coefficient is small and the diffusion is slower in the perpendicular to the 
edge, which in turn prevents the diffusion of the edge. However, in the flat areas, noise suppression is not sufficient, which even causes the false edges and generates the staircase effect.

In this paper, we mainly focus on solving the challenges mentioned in the previous remote sensing image denoising applications based on the introduced generalized TV model. In order to overcome shortcomings of TV denoising, this paper proposes a Sobel-TV model algorithm to denoise for remote sensing images. The algorithm extracts the image edge through using Sobel algorithm [10-11], then uses TV algorithm to remove image noise according to image edge information. The algorithm not only suppresses image noise, but also preserves the image edge and texture information, which is to enhance signal to noise ratio and improve image quality.

The rest of this paper is organized as follows. Section 2 describes the proposed method. Section 3 describes the algorithm implementation Section 4 analyses and compares the experimental results, followed by the conclusion in Section 5 .

\section{Literature Review}

A During the past three decades, a variety of methods have been proposed for image denoising in the image pre-processing and computer vision communities. Although seemingly very different, they all share the same property: to keep the meaningful edges and remove less meaningful ones. The existing image denoising work can be summarized as follows:

\subsection{Gaussian Filter}

By Riesz's theorem, Gaussian low pass filter is the filter which is impulse responsive, image isotropic linear filtering boils down to a convolution of the image by a linear radial kernel [12]. The smoothing requirement is usually expressed by the positivity of the kernel. The paradigm of such kernels is, of course, the Gaussian .In that case, has standard deviation h. So Gaussian is smoothing filter in the $2 \mathrm{D}$ convolution operation that is used to remove noise and blur for image.

\subsection{Mean Filter}

Mean Filter [13] is a simple linear filter, intuitive and easy to implement method of smoothing images, i.e. reducing the amount of intensity variation between one pixel and the next. It is often used to reduce noise in the image. The main idea of mean filtering is simply to replace each pixel value in an image with the mean (average) value of its neighbors, including itself. This has the effect of eliminating pixel values which are unrepresentative of their surroundings. Mean filtering is usually thought of as a convolution filter. Like other convolutions it is based around a kernel, which represents the shape and size of the neighborhood to be sampled when calculating the mean.

\subsection{Median Filter}

Median filter [14-15] is the non-linear filter which changes the image intensity mean value if the spatial noise distribution in the image is not symmetrical within the window, somewhat like the mean filter. However, it often does a better job than the mean filter of preserving useful detail in the image. Median filter is the variance of the intensities in the image. Median filter is a spatial filtering operation, so it uses a 2-D mask that is applied to each pixel in the 
input image. To apply the mask means to centre it in a pixel, evaluating the covered pixel brightness and determining which brightness value is the median value.

\subsection{Wiener Filter}

The Wiener filtering [16-17] is optimal in terms of the mean square error. In other words, it minimizes the overall mean square error in the process of inverse filtering and noise smoothing. The Wiener filtering is a linear estimation of the original image. The approach is based on a stochastic framework. Adaptive Wiener Filter (AWF) changes its behavior based on the statistical characteristics of the image inside the filter window. Adaptive filter performance is usually superior to non-adaptive counterparts. But the improved performance is at the cost of added filter complexity. Mean and variance are two important statistical measures using which adaptive filters can be designed.

\section{Methodology}

The Total Variation minimization was introduced by Rudin, Osher and Fatemi [3-4, 18]. Unlike a conventional low-pass filter, TV denoising is defined in terms of an optimization problem. The output of the TV denoising 'Filter' is obtained by minimizing a particular cost function. The TV (total variation) method makes the image restoration problem transformed into a functional extreme problem through introducing the energy function, which is widely used in image denoising. According to this principle, reducing the total variation of the signal subject to it being a close match to the original signal, removes unwanted detail whilst preserving important details such as edges.

When an image $u_{0}$ is corrupted by additive noise $n$, the result becomes a noisy image $u$ which can be represented by:

$$
u=J * u_{0}+n
$$

where $J$ is the Gaussian convolution operator. Set $J=I$ (Unit matrix), and $n$ is assumed to follow a Gaussian distribution with a zero mean and a variance of $\sigma^{2}$, hence equation (1) is simplified:

$$
u=u_{0}+n
$$

The TV denoising model, as described in [3] and [4], is denoted by

$$
T V(u)=\int_{S}|\nabla u| d x d y+\frac{\lambda}{2} \int_{S}\left(u-u_{0}\right)^{2} d x d y
$$

Here, $\mathrm{S}$ is the support area of the image, $u_{0} \in L^{2}(S)$, and have Lipschitz continuous boundary; $u \in L^{2}(S)$ is the observation image with noise; $\int_{S}|\nabla u| d x d y$ is the regularization term, and $\lambda$ is the regularization parameter, which plays a balancing role in the regularization and approximation term. $\nabla u$ denotes the gradient of $\mathrm{u}_{0}$, and $|\nabla u|$ is the modulus of $\nabla u$. The first one of the right of equation (3), called regex, is TV norm of image $u$, which relies on the edge of the image. Regular in the TV model has played a very important role. It allows that the image has non-continuous part, but does not allow that the image has oscillations part, so it can remove the noise of the image. The second of the right of equation (3) is approximation 
term, which controls the different between the image $u$ and $u_{0}$ the observation image. Minimizing the object function of (3) with respect to $\mathrm{u} 0$, as in (2), (3), we obtain (4) for $u_{0}$ the following:

$$
\left\{\begin{array}{l}
u_{t}=\frac{\partial T V(u)}{\partial u}=\operatorname{div}\left(\frac{\nabla u}{|\nabla u|}\right)+\lambda\left(u_{0}-u_{t}\right) \\
u(x, y, 0)=u_{0}
\end{array}\right.
$$

In Eq. (4), div (.) is the divergence operator. $u_{t}=u(x, y, t), \mathrm{t}$ is the number of iterations. $(x, y) \in S, t>0, \nabla u$ is the gradient of image $u$.The TV model is a piecewise continuous function in the bounded variation space. Hence, the model has good removal effect for the repeating patterns of small-scale details and noise, but it is likely to cause loss of details for the rich texture image.

In (4), fidelity approximation $\lambda\left(u_{0}-u_{t}\right)$ keeps the original image features and reduces image distortion effect, while diffusion term $\operatorname{div}\left(\frac{\nabla u}{|\nabla u|}\right)$ removes noise of the image. When diffusion term removes noise of the image, it will smooth the whole image by the same degree of denosing, which leads to lose the small characteristics and blur the image edge. To solve this problem, in (4) introduce a guide function $m(x, y)$ is introduced to improve Eq. 4 as follows:

$$
\left\{\begin{array}{l}
u_{t}=\operatorname{div}\left(\frac{\nabla u}{|\nabla u|}\right) m(x, y)+\lambda\left(u_{0}-u_{t}\right) \\
u(x, y, 0)=u_{0}
\end{array}\right.
$$

The values of the function $\mathrm{m}(\mathrm{x}, \mathrm{y})$ are low in places where the amount of change in gray levels is high and vice versa. The value of $\mathrm{m}(\mathrm{x}, \mathrm{y})$ is the bigger in the slower gray value of the place. Therefore, the function can selectively denoise or smooth image according to the amount of change of gray-scale values in the each part of the image. From the above discussion, the function can be expressed as follows:

$$
m(x, y)=\frac{1}{1+\left(\frac{g(x, y)}{T}\right)^{2}}
$$

In Eq. (6), $m(x, y) \in R^{+}$and $0<m(x, y)<1 ; g(x, y)$ is the edge detection value of the point $(\mathrm{x}, \mathrm{y})$ in the image; $\mathrm{T}$ is a prior threshold value and its value may be changed according to the demand of image processing, .

Through edge detection, the image $u_{0}$ can be transformed into $g(x, y)$. The mathematical model of edge detection has first order differential operators and second order differential operators. Since the computation of the second-order differential operator is very complex, we choice first-order differential operators, which is more flexible. In literature, there are two first order differential which are used for edge detection; Prewitt operator and Sobel Operator. 
Prewitt operator is very sensitive to noise and remove image noise, but it maintains worse edges in the image than sobel operator. Sobel operator weights the impact of the image pixel location, which can reduce the degree of edge blur, and hence it has better effect.

The Sobel operator uses two $3 \times 3$ kernels which are convolved with the original image to calculate approximations of the derivatives-one for horizontal changes, and one for vertical. If we define U0 as the source image, and Gx and Gy are two images which at each point contain the horizontal and vertical derivative approximations, the computations are as follows:

$$
G_{x}=\left[\begin{array}{ccc}
-1 & 0 & +1 \\
-2 & 0 & +2 \\
-1 & 0 & +1
\end{array}\right] * U_{0} \quad \text { and } \quad G_{y}=\left[\begin{array}{ccc}
+1 & +2 & +1 \\
0 & 0 & 0 \\
-1 & -2 & -1
\end{array}\right] * U_{0}
$$

Where $*$ here denotes the 2-dimensional convolution operation.

Since the Sobel kernels can be decomposed as the products of an averaging and a differentiation kernel, they compute the gradient with smoothing.

\section{Algorithm Implementation}

A Sobel-TV algorithm for image denoising can be achieved by employing a sobel operator to detect image edges, then invoking TV function to remove image noise. The algorithm can be implemented as follows:

STEP1. Choose Sobel operator to detect the edges in the image under consideration

$$
g(i, j)=|f(i+1, j)-f(i-1, j)|+|f(i, j+1)-f(i, j-1)|
$$

STEP2. Using (9) remove image noise according to the image edge information.

$$
f^{n+1}(i, j)=\left[\operatorname{div}^{n}\left(\frac{\nabla f}{|\nabla f|_{\beta}}\right) g(i, j)+\lambda^{n}\left(f^{0}(i, j)-f^{n}(i, j)\right)\right] \Delta t+f^{n}(i, j)
$$

Where $f^{n}(i, j)$ is the result of $\mathrm{n}$ iterations, $f^{n+1}(i, j)$ is the result of $(\mathrm{n}+1)$ iterations. $\Delta t$ is the time step length. $\lambda^{n}$ is the parameter of the regular .In order to avoid the expression $\frac{\nabla f}{|\nabla f|}$ is not zero, we have introduced the parameter by defining

$$
|\nabla f|_{\beta}^{2}=|\nabla f|^{2}+\beta
$$

According to divergence, it arrives as follows.

$$
\operatorname{div}^{n}\left(\frac{\nabla f}{|\nabla f|_{\beta}}\right)=\frac{f_{x x}^{n}\left(f_{y}^{n}\right)^{2}+f_{y y}^{n}\left(f_{x}^{n}\right)^{2}-2 f_{x}^{n} f_{y}^{n} f_{x y}^{n}}{\left(\left(f_{x}^{n}\right)^{2}+\left(f_{y}^{n}\right)^{2}+\beta^{2}\right)^{3 / 2}}
$$


where $f_{x}$ the first derivatives in the $\mathrm{x}$ direction; $f_{y}$ the first derivatives in the $\mathrm{y}$ direction; $f_{x x}$ the second derivatives in the $\mathrm{x}$ direction $f_{y y}$ the second derivatives in the $\mathrm{y}$ direction; $f_{x y}$ the second derivatives for $f_{x}$. The boundary conditions are:

$$
\left\{\begin{array}{l}
f^{n}(0, j)=f^{n}(1, j) \\
f^{n}(N, j)=f^{n}(N-1, j) \\
f^{n}(i, 0)=f^{n}(1, N)=f^{n}(i, N-1)
\end{array}\right.
$$

The scale parameter formula is as follows:

$$
\lambda^{n}=-\frac{1}{2 \sigma^{2}}\left[\sum_{i j}\left(\sqrt{\left(\left(f_{x}^{n}\right)^{2}+\left(f_{y}^{n}\right)^{2}\right)^{2}}-\frac{\left(f_{x}^{0}\right)\left(f_{y}^{n}\right)+\left(f_{y}^{0}\right)\left(f_{x}^{n}\right)}{\sqrt{\left(f_{x}^{n}\right)^{2}+\left(f_{y}^{n}\right)^{2}}}\right)\right]
$$

In this paper, the parameter is set as follows:

$\Delta t=0.2 ; \beta=1 ; \lambda^{n} \approx 0.5$.

\section{Numerical Experiments}

The proposed model was tested on a number of remote sensing images which were collected for this purpose. All numerical experiments were performed on 64-bit Windows 7 on a desktop with an Intel Core i3 CPU at $2.27 \mathrm{GHz}$ and 4GB memory. In order to estimate the performance of different methods, we use Signal to Noise Ratio (SNR) as the image quality measure which is defined as follows,

$$
S N R=10 \times \log _{10}\left(\frac{\sum_{i=1}^{n} \sum_{j=1}^{n}\left(u^{0}(i, j)-\operatorname{mean}\left(u^{0}\right)\right)^{2}}{\sum_{i=1}^{n} \sum_{j=1}^{n}\left(u^{*}(i, j)-u^{0}(i, j)\right)^{2}}\right)
$$

In Eq. $18, \mathrm{u} 0$ is the original image, $\mathrm{u}^{*}$ is the mean intensity value of the restored image $\mathrm{u}$. We also adopt the structure similarity index measure (SSIM) to measure the similarity between two images, which is defined as

$$
\operatorname{SSIM}\left(u^{*}, u^{0}\right)=\frac{\left(2 \mu_{u^{*}} \mu_{u^{0}}+c_{1}\right)\left(2 \sigma_{u^{*} u^{0}}+c_{2}\right)}{\left(\mu_{u^{*}}^{2}+\mu_{u^{0}}^{2}+c_{1}\right)\left(\sigma_{u^{*}}^{2}+\sigma_{u^{0}}^{2}+c_{2}\right)}
$$

Where $\mu_{u}$ is the average of $u^{0}, \sigma^{2}$ is the variance of $u^{0}, \sigma_{u^{*} u^{0}}$ is the covariance of $u^{*}$ and and $u^{0}$ are two constants to avoid instability.

\subsection{Parameter Values [19-21]}

In order to obtain the number of iterations in Eq.13, we add Gaussian noise with the standard deviation $\sigma=10$ and Salt-pepper noise with the density $\mathrm{D}=0.1 \%$ to the image Building 1. Figures 1(a) and Figures 1 (b) show the relationship between SNR or SSIM and the number of iterations. The results show that the SNR and SSIM is the maximum value when the number of iterations is about 100.Therefore, the number of iterations in Eq. (11) chooses 100 . 


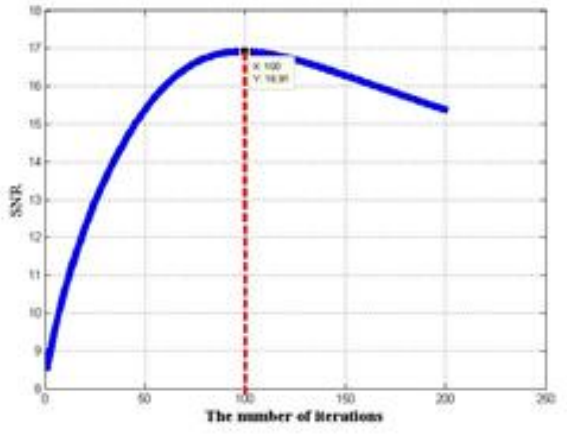

(a)

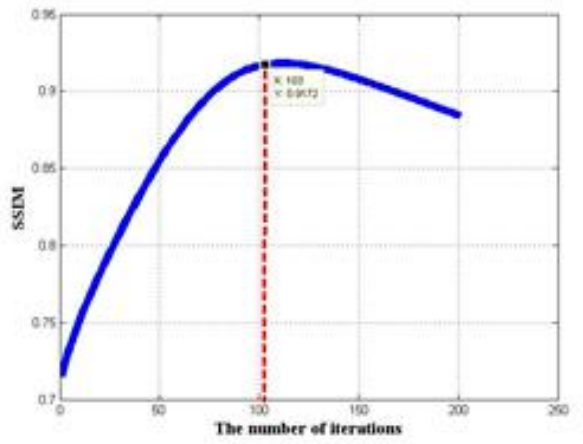

(b)

Figure 1. (a) The Relationship between SNR and the Number of Iterations Figure 1 (b) the Relationship between SSIM and the Number of Iterations

\subsection{Experiments on Simulated Noisy Images}

To validate and compare the proposed method, we perform the simulation experiments on different datasets. These datasets contain both the noisy and noise-free images of $256 \times 256$ pixels. The noisy images are simulated by contaminating the original image with additive Gaussian noise with the standard deviation $\sigma=15$ and Salt-pepper noise with the density $\mathrm{D}=$ $0.1 \%$. When the noise is simulated, the performance of the different denoising techniques can be quantitatively described by the SNR and the SSIM.

The noisy images are shown in the second column of Figures 2. The result using mean filter and median filter algorithm are shown in the third and the fourth column of Figures 2. The result using the Sobel-TV algorithm is shown in the fifth of Figures 2. Let us comment on them briefly. The mean filter method removes the noise and retains the important features of the image, but the image is very blurred. The median filter method also removes the noise, but it loss the detail and structure of the image. The proposed Sobel-TV method not only removes the noise but also preserved well the contours, texture and details. The testing results demonstrate that the Sobel-TV algorithm for de-noising image is better than the other algorithm.

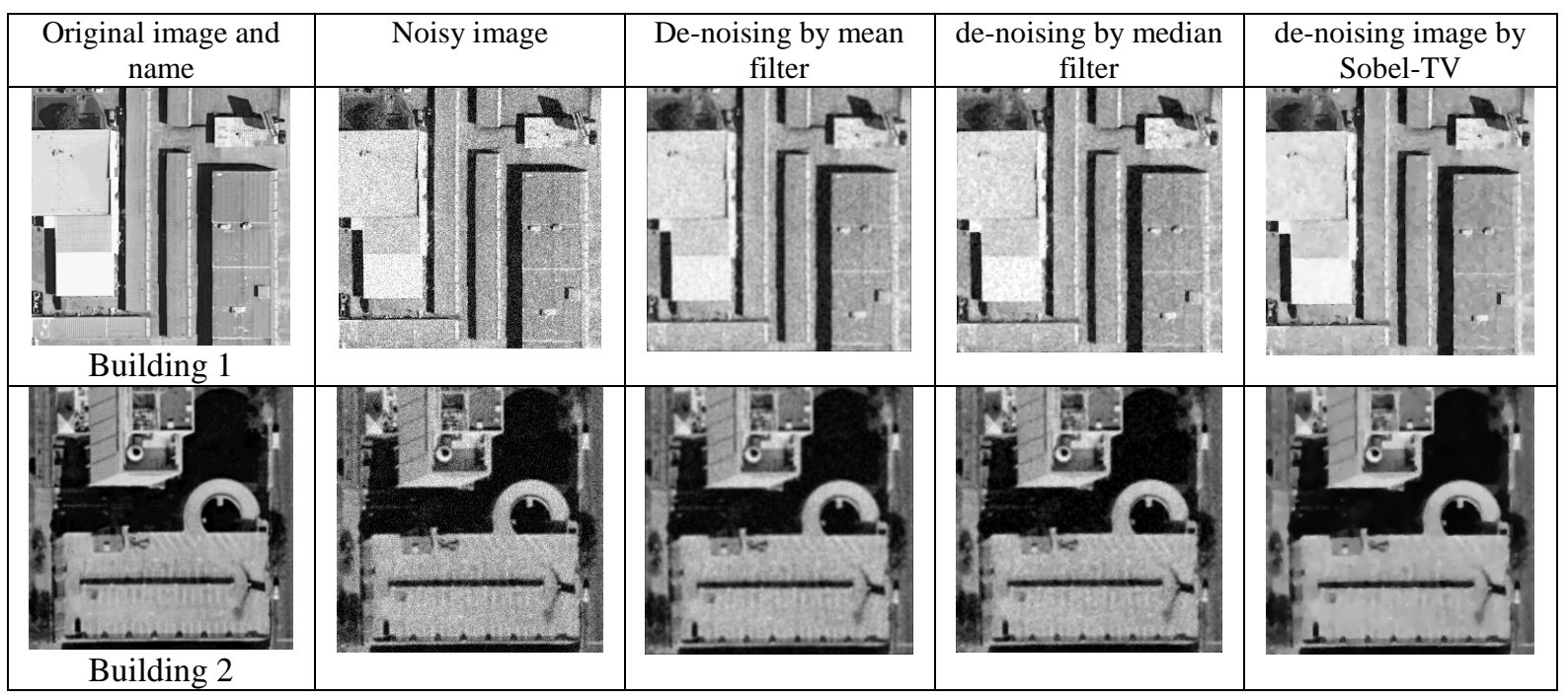


International Journal of Signal Processing, Image Processing and Pattern Recognition Vol.8, No.3 (2015)

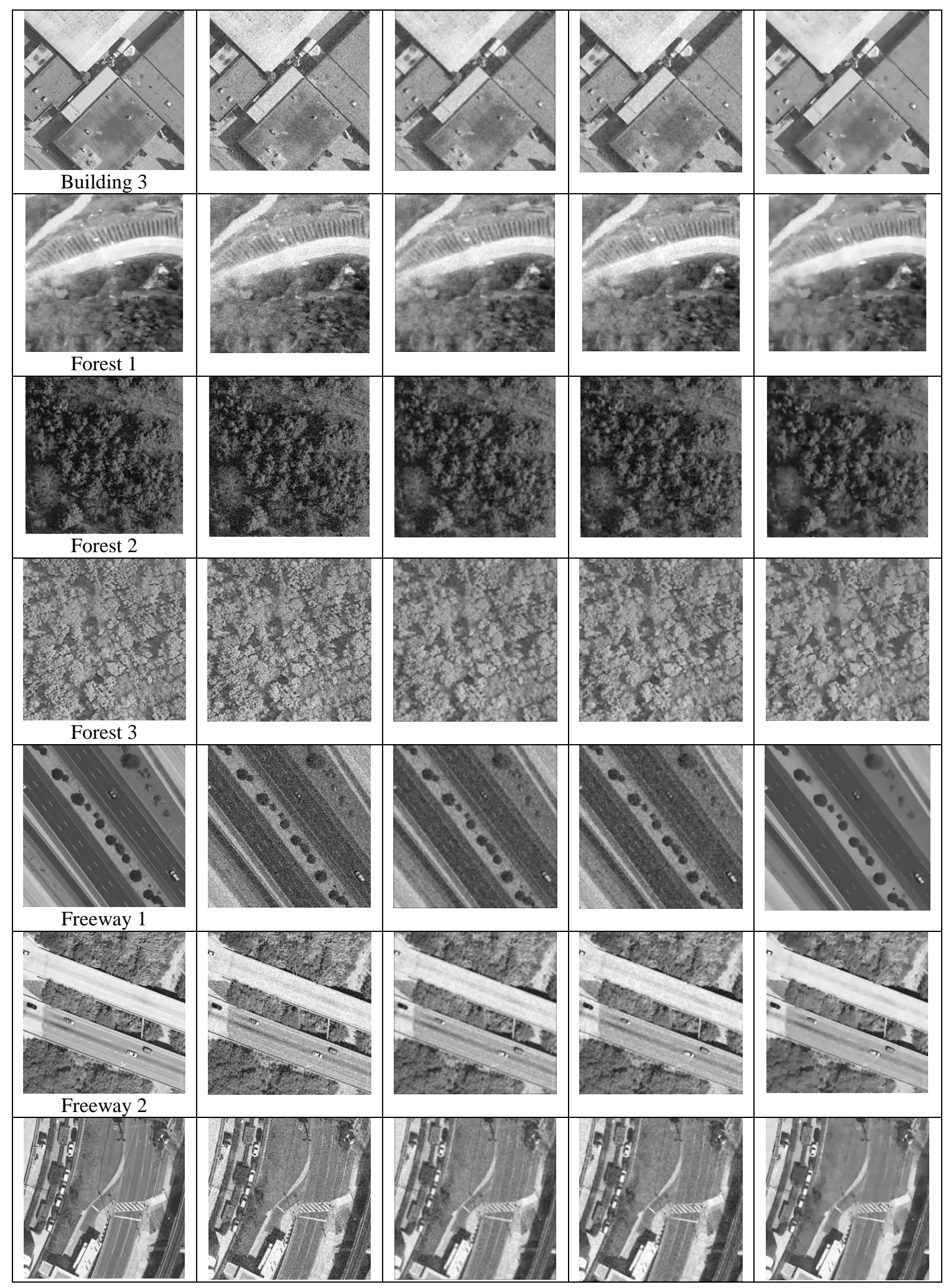




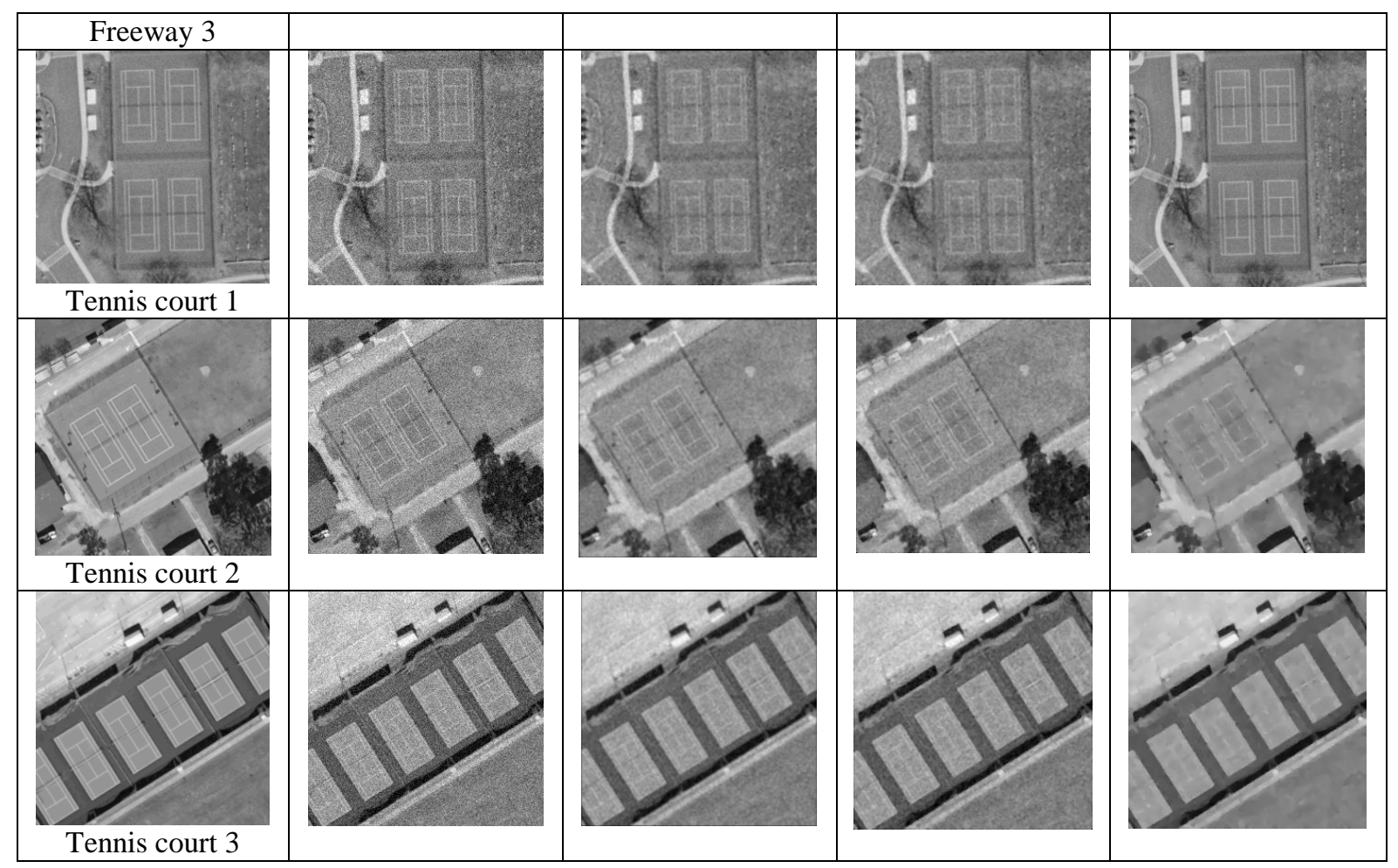

Figure 2. Noise in Different Images was Removed by Mean Filter, Median Filter, and by the Proposed Method

To further validate the proposed Sobel-TV method, the signal to noise measure (SNR) and the structural similarity index (SSIM) was used to evaluate the performance of the proposed algorithm. Table1 and Table 2 shows the SNR and SSIM values obtained in the visualization domain for a dataset of 12 remote sensing images. Comparing the filtering methods with respect to the error measures, the best SNR and SSIM value for Sobel-TV are obtained. With respect to the SNR and SSIM value, we also find quantitatively that our method yields better denoising results.

Table 1. Comparison of the SNR Values

\begin{tabular}{lllll}
\hline & Noise image & Median & Mean(3*3) & TV-SOBEL \\
\hline Building1 & 10.9426 & 13.4961 & 11.7269 & 16.1958 \\
Building2 & 12.1656 & 17.2582 & 17.0392 & 18.4247 \\
Building3 & 8.3211 & 12.7249 & 11.3836 & 14.4952 \\
Forest1 & 9.2697 & 15.4340 & 14.9600 & 16.7986 \\
Forest2 & 5.2788 & 6.8891 & 7.2620 & 7.3351 \\
Forest3 & 5.7761 & 6.2229 & 6.1300 & 6.8658 \\
Freeway1 & 5.7499 & 9.9445 & 9.6020 & 11.6594 \\
Freeway2 & 9.6884 & 11.2917 & 10.7165 & 12.3447 \\
Freeway3 & 8.8087 & 11.4341 & 10.6251 & 12.9271 \\
Tenniscourt1 & 2.1925 & 7.9427 & 7.6747 & 9.4116 \\
Tenniscourt2 & 6.7730 & 12.3883 & 11.9155 & 14.0591 \\
Tenniscourt3 & 8.0494 & 13.9591 & 12.9916 & 16.1476 \\
\hline
\end{tabular}


Table 2. Comparison of the SSIM Values

\begin{tabular}{lllll}
\hline & Noise image & Median & Mean $(3 * 3)$ & TV-SOBEL \\
\hline Building1 & 0.5641 & 0.7137 & 0.7336 & 0.8317 \\
Building2 & 0.5483 & 0.7943 & 0.8238 & 0.8465 \\
Building3 & 0.4610 & 0.7031 & 0.7483 & 0.8099 \\
Forest1 & 0.4715 & 0.7831 & 0.8013 & 0.8410 \\
Forest2 & 0.6997 & 0.6575 & 0.6812 & 0.6959 \\
Forest3 & 0.5634 & 0.7109 & 0.7213 & 0.8144 \\
Freeway1 & 0.4548 & 0.6765 & 0.7254 & 0.8044 \\
Freeway2 & 0.6399 & 0.7287 & 0.7549 & 0.7639 \\
Freeway3 & 0.5919 & 0.7465 & 0.7754 & 0.8024 \\
Tenniscourt1 & 0.4207 & 0.6959 & 0.6846 & 0.7511 \\
Tenniscourt2 & 0.4170 & 0.7129 & 0.7710 & 0.8247 \\
Tenniscourt3 & 0.4367 & 0.7320 & 0.7836 & 0.8400 \\
\hline
\end{tabular}

From the Figures 2 and the numerical results in the table(Table1 Table2), we can draws the following conclusions : the proposed Sobel-TV method have a better visualization and achieve the highest SNR and SSIM values, it is evident that the proposed method is effective in both removing image noise and maintaining detail information.

\section{Conclusion}

Traditional TV algorithm diffuses in the direction to edge on the flat area, which preserves sharp edges and removes the noise. But it will cause that image noise suppression is not sufficient, even show a false boundary. In this paper, an improved algorithm on TV remote sensing image is proposed. The algorithm utilizes the sobel operator to detect the spatial gradient, and then suppresses the spatial gradient by thresholding, which solve the TV defaults such as unsufficient noise suppression, the false edges and ladder effects. And then combining with pixel gradient, the method removes the noise of the images and preserves the edges and texture details information of the images effectively. We have also presented some promising evidence for our methods on a real remote sensing image dataset. Compared with traditional image denoising algorithm, experimental results show that the algorithm has better edge-protection and less residual noise on SNR and SSIM, which is consistent with the visual effect illustrated by denoised images. In the general, remote sensing image is bigger than the other's optical image and the algorithm's complexity proposed by this paper is very high, so the denoising process is time consuming. In the future, we will speed up the denoising algorithm by GPU accelerate, which is satisfied the actual demand for remote sensing image processing.

\section{References}

[1] L. R. Gao, B. Zhang and X. Zhang, "Study on the Method for Estimating the Noise in Remote Sensing Images Based on Local Standard Deviations", Journal of Remote Sensing, vol. 11, no. 2, (2007), pp. 201-208.

[2] S. S. Al-amri, N. V. Kalyankar and S. D. A. Khamitkar, "Comparative study of removal noise from remote sensing image", International Journal of Computer Science Issues, vol. 7, no. 1, (2010) January, pp. 32-36.

[3] L. Rudin, S. Osher, E. Ftemi, "Nonlinear total variation based noise removal algorithm", Phys. D, vol. 60, (1992), pp. 259-268.

[4] L. Rudin and S. Osher, "Total variation based image restoration with free local constraints", In: Proceedings of 1st IEEE Int. Conf. on Image Process., Austin, (1994), pp. 31-35.

[5] A. Chambolle and P.-L. Lions, "Image recovery via total variation minimization and related problems", Numer. Math., (1997), pp. 76, 167-188. 
[6] T. F. Chan, S. Esedoglu, F. Park and A. Yip, "Recent developments in total variation image restoration", In: The Handbook of Mathematical Models in Computer Vision, Springer, Berlin, (2005).

[7] S. Durand and M. Nikolova, "Denoising of frame coefficients using 11 data fidelity term and edge preserving regularization", Multiscale Model. Simul., vol. 6, no. 2, (2007) June, pp. 547-576.

[8] D. Q. Chen, H. Zhang and L. Z. Cheng, "A fast fixed point algorithm for total variation deblurring and segmentation”, J. Math. Imag. Vis., 2011.doi:10.1007/s10851-011-0298-7, vol. 43, (2012) July, pp. 167-179.

[9] Y. Lou, X. Zhang, S. Osher and A. Bertozzi, "Image recovery via nonlocal operators", J. Sci. Comput. vol. 42, no. 2, (2010), pp. 185-197.

[10] R. Gonzalez and R. Woods, "Digital Image Processing", Addison Wesley, (1992), pp. 414-428.

[11] A. Hast, "Simple filter design for first and second order derivatives by a double filtering approach", Pattern Recognition Letters, vol. 42, no. 1, (2014) June, pp. 65-71.

[12] R. A. Haddad and A. N. Akansu, "A Class of Fast Gaussian Binomial Filters for Speech and Image Processing", IEEE Transactions on Acoustics, Speech and Signal Processing, vol. 39, (1991) March, pp. 723 727.

[13] R. Boyle and R. Thomas, "Computer Vision: A First Course, Blackwell Scientific Publications”, (1988), pp. $32-34$.

[14] T. Huang, G. Yang and G. Tang, "A fast two-dimensional median filtering algorithm”, IEEE Trans. Acoust. , Speech, Signal Processing, vol. 27, no. 1, (1979), pp. 13-18.

[15] E. Arias-Castro and D. L. Donoho, "Does median filtering truly preserve edges better than linear filtering?", Annals of Statistics, vol. 37, no. 3, (2009) April, pp. 1172-1206.

[16] J. S. Lim and A. V. Oppenheim, "Enhancement and bandwidth compression of noisy speech", Proc. IEEE, vol.67, no. 12, (19709) December, pp. 1586-1604.

[17] J. D. Chen, B. Jacob, Y. T. Huang and D. Simon, "New Insights Into the Noise Reduction Wiener Filter", IEEE Trans. Audio, Speech, and Language Processing, vol. 14, no. 4, (2006) July, pp. 1218-1234.

[18] O. V. Michailovich, "An iterative shrinkage approach to total-variation image restoration", IEEE Trans. Image Process., vol. 20, no. 5, (2011), May, pp. 1281-1299.

[19] M. Felsberg, "Autocorrelation-driven diffusion filtering”, IEEE Trans. Image Process., vol. 20, no. 20, July (2011), pp. 1797-1806.

[20] F. Astrom, M. Felsberg, G. Baravdish and C. Lundstrom, "Targeted Iterative Filtering. Fourth International Conference on Scale Space and Variational Methods in Computer Vision”, vol. 7893, (2013) June, pp. 1-11.

[21] P. Liu, H. Fang, G. Q. Li and Z. W. Liu, "Remote-Sensing Image Denoising Using Partial Differential Equations and Auxiliary Image As Priors", Multiscale Model Simul., vol. 4, no. 2, (2012), May, pp. 490-530. 
International Journal of Signal Processing, Image Processing and Pattern Recognition Vol.8, No.3 (2015) 